\title{
Characteristic Improvement of a Novel Current Observer Based Induction Motor Speed Sensorless System
}

\author{
Mineo Tsuji*, Fujin Xu*, Yasutaka Tsuruda*,Shin-ichi Hamasaki*, and Eiji Yamada* \\ * Department of Electrical and Electronic Engineering, Nagasaki University, Bunkyo-machi 1-14, \\ Nagasaki 852-8521, E-mail: mineo@nagasaki-u.ac.jp, (JAPAN)
}

\begin{abstract}
We have proposed a current observer-based induction motor speed sensorless vector control system which is composed in a rotating reference frame. The characteristic improvement of this system is discussed by taking into account iron loss, stator resistance identification, current low-pass filter and speed estimation low-pass filter. The stability of the system has been investigated by the trajectories of poles and zeros. The effectiveness of the proposed system has been investigated by digital simulation and experimentation.
\end{abstract}

Index Terms: sensorless vector control, current observer, stability analysis, parameter identification, iron loss

\section{INTRODUCTION}

Model reference adaptive system (MRAS) based speed sensorless vector control of induction motor has been studied in many papers [1][2]. In these papers, the flux or current observer is composed in a stationary reference frame in which the variables are AC quantities. A survey of sensorless vector control of induction motor is described the conference of Speedam [3]. On the other hand, we have proposed the speed sensorless vector control systems in a synchronously rotating reference frame [4][5][6]. In our methods, the variables are DC quantities and the linear state equations are obtained easily. In this paper, the characteristic improvement of a current observer-based speed sensorless vector control system is discussed by taking into account iron loss, stator resistance identification, current low-pass filter and speed estimation low-pass filter. The stability of the system has been investigated by the trajectories of poles and zeros, which are computed from the linear model for small changes of state variables. The effectiveness of the proposed system has been studied analytically and experimentally.

\section{PROPOSED METHOD}

Figure 1 shows the proposed speed sensorless vector control system [6]. In this system, the $d-q$ axis is chosen such that the $d$ axis is on the computed rotor flux vector and the iron loss is taken into account.

The current observer is expressed as

$$
\begin{aligned}
p \hat{i}_{s d}= & \frac{1}{\sigma L_{s}}\left\{e_{s d}^{*}-\left(R_{s}^{*}+\frac{M^{2}}{\tau_{r}^{*} L_{r}}\right) \hat{i}_{s d}+\sigma L_{s} \omega^{*} \hat{i}_{s q}\right. \\
& \left.+\frac{M}{\tau_{r}^{*} L_{r}} \hat{\psi}_{r d}+\frac{M l_{r}}{L_{r}} \omega^{*} \hat{i}_{c q}\right\}+K_{a}\left(i_{s d}-\hat{i}_{s d}\right) \\
p \hat{i}_{s q}= & \frac{1}{\sigma L_{s}}\left(e_{s q}^{*}-R_{s}^{*} \hat{i}_{s q}-\omega^{*} \sigma L_{s} \hat{i}_{s d}-\frac{\omega^{*} M}{L_{r}} \hat{\psi}_{r d}\right)
\end{aligned}
$$

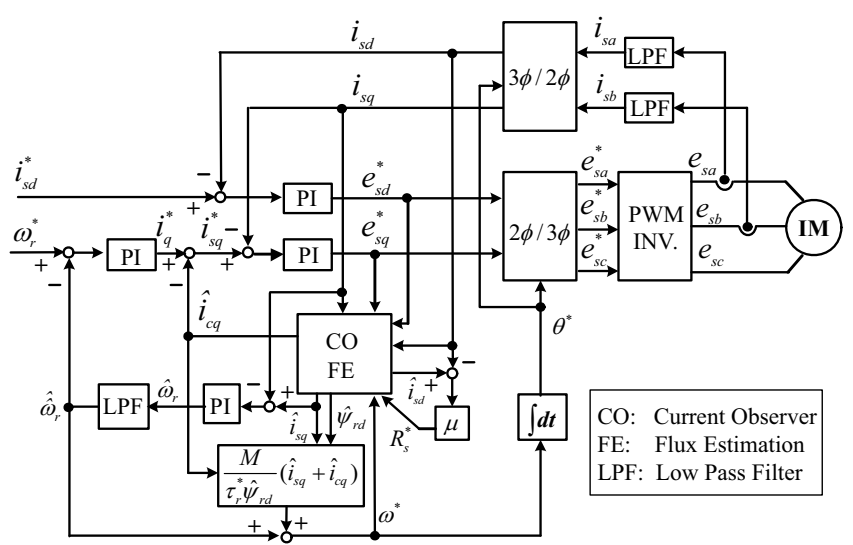

Fig.1. Proposed sensorless vector control system.

where, $p=d / d t, \tau_{r}^{*}=L_{r} / R_{r}^{*}$ and $K_{a}$ is observer gain.

The $d$-axis rotor flux is computed as

$$
p \hat{\psi}_{r d}=\frac{M}{\tau_{r}^{*}} \hat{i}_{s d}-\frac{1}{\tau_{r}^{*}} \hat{\psi}_{r d}
$$

The estimated iron loss current is expressed in the following equation.

$$
\hat{i}_{c q}=-\frac{\omega^{*} M}{R_{m}^{*} L_{r}}\left(\hat{\psi}_{r d}+l_{r} \hat{i}_{s d}\right)
$$

where, $R_{m}^{*}$ is the iron loss resistance connected in parallel with mutual inductance. From the viewpoint of MRAS theory, the speed estimation is realized by

$$
\hat{\omega}_{r}=K_{c}\left(1+\frac{1}{T_{c} p}\right)\left(\hat{i}_{s q}-i_{s q}\right)
$$

In odder to reduce the ripple of $\hat{\omega}_{r}$, a low pass filter having cut-off angular frequency $\omega_{L P F}$ is introduced.

$$
p \hat{\hat{\omega}}_{r}=\omega_{L P F}\left(\hat{\omega}_{r}-\hat{\hat{\omega}}_{r}\right)
$$

Compensating the $q$-axis iron loss current, the estimated angular frequency of flux $\omega^{*}$ is calculated by

$$
\omega^{*}=p \theta^{*}=\hat{\hat{\omega}}_{r}+\frac{M}{\tau_{r}^{*} \hat{\psi}_{r d}}\left(\hat{i}_{s q}+\hat{i}_{c q}\right)
$$

Using the $d$-axis current error, stator resistance identification is done by

$$
p R_{s}^{*}=\mu\left(\hat{i}_{s d}-i_{s d}\right)
$$

\section{STABILITY ANALYSIS}

A linear model of induction motor taking into account iron loss is expressed as follows by selecting the $d-q$ axis that 
rotates with $\theta^{*}$ :

$$
p \Delta \boldsymbol{x}_{s}=\boldsymbol{A}_{s} \Delta \boldsymbol{x}_{s}+\boldsymbol{B}_{s} \Delta \boldsymbol{u}_{s}+\boldsymbol{B}_{l} \Delta T_{L}
$$

where,

$$
\begin{aligned}
\Delta \boldsymbol{x}_{s} & =\left[\Delta i_{s d}, \Delta i_{s q}, \Delta \psi_{r d}, \Delta \psi_{r q}, \Delta i_{m d}, \Delta i_{m q}, \Delta \omega_{r}\right]^{T} \\
\Delta \boldsymbol{u}_{s} & =\left[\Delta e_{s d}, \Delta e_{s q}, \Delta \omega^{*}\right]^{T}
\end{aligned}
$$

A linear model of the control system which includes PI current and speed regulators is derived as follows:

$$
p \Delta \boldsymbol{z}=\boldsymbol{A}_{x} \Delta \boldsymbol{x}_{s}+\boldsymbol{A}_{z} \Delta \boldsymbol{z}+\boldsymbol{B}_{z} \Delta \boldsymbol{u}_{s}+\boldsymbol{B}_{r} \Delta \omega_{r}^{*}+\boldsymbol{B}_{c} \Delta \hat{i}_{c q}
$$

where,

$$
\begin{aligned}
& \Delta \boldsymbol{z}=\left[\Delta e_{c}, \Delta e_{s}, \Delta e_{v d}, \Delta e_{v q}, \Delta \hat{i}_{s d}, \Delta \hat{i}_{s q}, \Delta \hat{\psi}_{r d}, \Delta \hat{\hat{\omega}}_{r}\right]^{T} \\
& \Delta \boldsymbol{u}_{s}=\boldsymbol{F}_{x} \Delta \boldsymbol{x}_{s}+\boldsymbol{F}_{z} \Delta z+\boldsymbol{F}_{r} \Delta \omega_{r}^{*}+\boldsymbol{F}_{c} \Delta \hat{i}_{c q} \\
& \Delta \hat{i}_{c q}=\boldsymbol{C}_{z} \Delta \boldsymbol{z}
\end{aligned}
$$

From (9), (10), (11), and (12), the linear state equation of the whole system is described as

$$
\begin{aligned}
p\left[\begin{array}{c}
\Delta \boldsymbol{x}_{s} \\
\Delta \boldsymbol{z}
\end{array}\right] & =\left[\begin{array}{cc}
\boldsymbol{A}_{s}+\boldsymbol{B}_{s} \boldsymbol{F}_{x} & \boldsymbol{B}_{s}\left(\boldsymbol{F}_{z}+\boldsymbol{F}_{c} \boldsymbol{C}_{z}\right) \\
\boldsymbol{A}_{x}+\boldsymbol{B}_{z} \boldsymbol{F}_{x} & \boldsymbol{A}_{z}+\boldsymbol{B}_{c} \boldsymbol{C}_{z}+\boldsymbol{B}_{z}\left(\boldsymbol{F}_{z}+\boldsymbol{F}_{c} \boldsymbol{C}_{z}\right)
\end{array}\right]\left[\begin{array}{c}
\Delta \boldsymbol{x}_{s} \\
\Delta \boldsymbol{z}
\end{array}\right] \\
& +\left[\begin{array}{c}
\boldsymbol{B}_{s} \boldsymbol{F}_{r} \\
\boldsymbol{B}_{r}+\boldsymbol{B}_{z} \boldsymbol{F}_{r}
\end{array}\right] \Delta \omega_{r}^{*}+\left[\begin{array}{c}
\boldsymbol{B}_{l} \\
\mathbf{0}
\end{array}\right] \Delta T_{L}
\end{aligned}
$$

Stability analysis can be done by the trajectories of poles and zeros about the speed transfer function obtained by (13) as shown in Figs.2, 3 and 4. The speed estimation gain is changed in Fig.2. If the gain $K_{c}$ is small, the dominant pole is close to imaginary axis and the system becomes oscillating. Fig. 3 shows the trajectories when the current observer gain is changed from 0 to 500 . If the observer gain is 0 , the dominant pole is close to imaginary axis. In this paper we choose 250 as $K_{a}$ because the real part of pole is small. Fig. 4 shows the trajectories when the stator resistance in the controller is only changed from $0.8 \Omega$ to $3.0 \Omega$. The actual value of stator resistance is set to $1.6 \Omega$. When $K_{a}=0$ as shown in Fig. (a), the system is unstable when the stator resistance of controller is larger than $2.2 \Omega$ but it is improved by choosing $K_{a}=250$ as shown in (b).

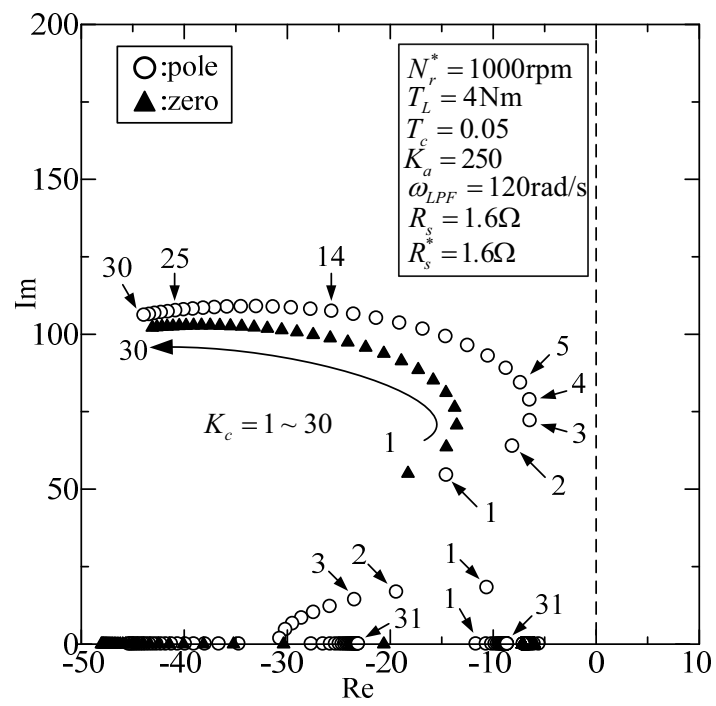

Fig.2. Trajectories of poles and zeros.

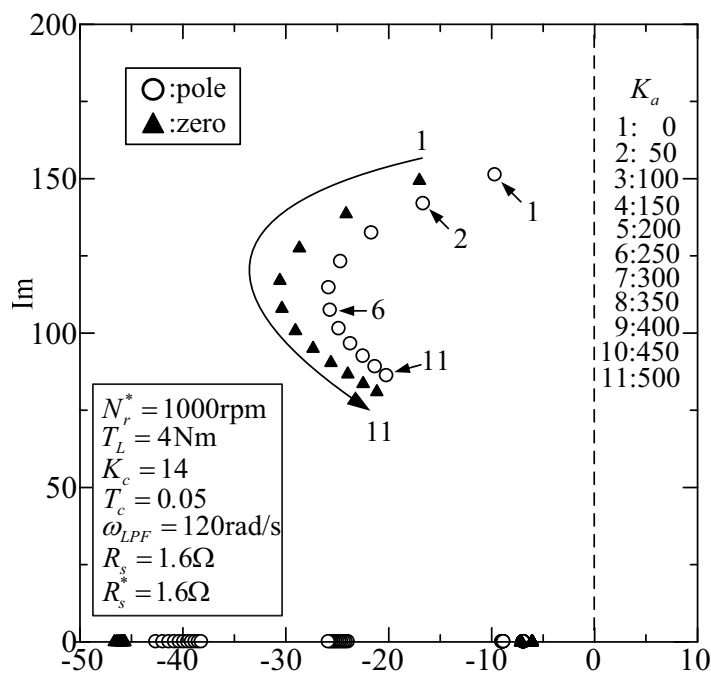

Fig. 3 Trajectories of poles and zeros with parameter observer gain.

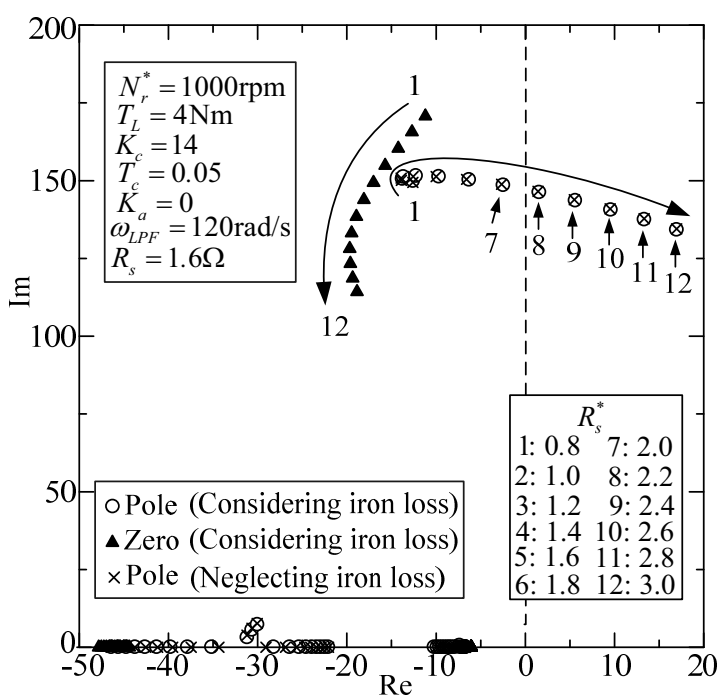

(a) $K_{a}=0$

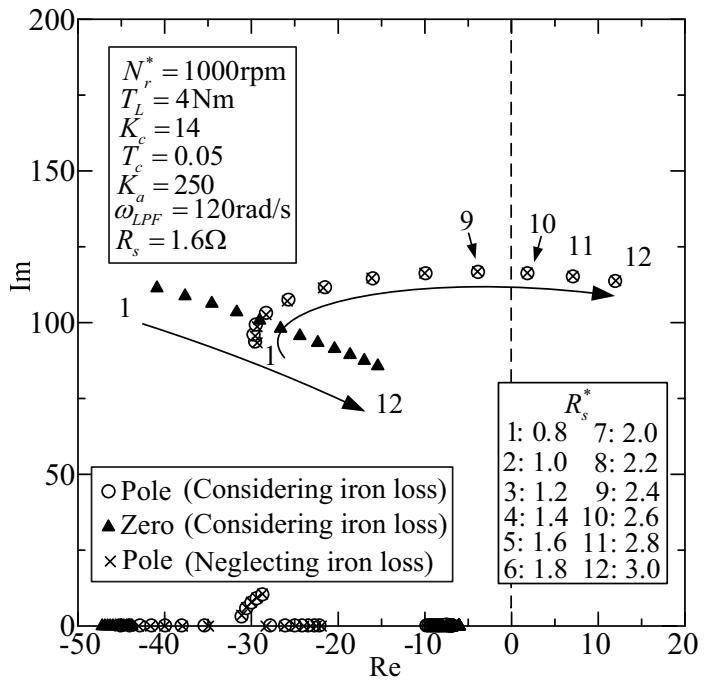

(b) $K_{a}=250$

Fig.4. Trajectories of poles and zeros with parameter stator resistance in the controller. 


\section{TRANSIENT CHARACTERISTICS}

The proposed control scheme was implemented by a DSP (TMS320C6713) and tested by PWM inverter-fed IM with switching frequency $5 \mathrm{kHz}$. We discuss the effect of control parameters on the transient characteristics by comparing experimental results with simulation results.

Figure 5 shows an experimental result when the speed command $N_{r}^{*}$ is changed form 1000rpm to 1100rpm and back to $1000 \mathrm{rpm}$. The step responses show the actual speed $N_{r}$, estimated speed $\hat{\hat{N}}_{r}$, phase current $i_{s}$ and estimated motor torque $\hat{\tau}_{e}$. In this case, detected stator currents are directly used to control. Pulsations of actual speed and estimated speed are observed. In order to solve this problem, we utilized a first order digital low pass filter whose cut-off frequency is $796 \mathrm{~Hz}$. All experimental results are obtained by using the low-pass filter except the case of Fig.5.

In order to compute transient responses of the proposed system in detail, we developed a simulation program, which includes the effects of PWM mechanism, non-ideal features of IGBT and diode, iron loss, dead time and digital control algorithm. In this case the IM is described by a stationary reference frame.

Because the deadtime and the non-ideal features of IGBT influence the output voltage of the inverter, we proposed a compensating algorithm[5]. Figures 6(a) and (b) show the simulation result and experimental result respectively when voltage compensation is not done. At high speed operation the effect of voltage compensation is small. The simulation results are in good agreement with the experimental ones.

In Fig.7, speed estimation gain $K_{c}$ is set to 2 . The overshoot of actual speed is large and large difference between actual speed and estimated one is observed. This result is predicted the trajectories of the poles and zeros shown in Fig.2. Close agreement between simulated and experimental values is obtained.

In Fig.8, speed estimation gain $K_{c}$ is set to 25 . The high frequency pulsation of estimated speed and torque is observed in experimental result, but it is not seen in the simulation result. So the reason of the pulsation may be some noise.

In Fig.9, current observer gain $K_{a}$ is set to 0. Large oscillation is observed in the experimental result. However, this oscillation is not confirmed by the simulation. When $K_{a}$ is zero, the dominant pole is close to imaginary axis as shown in Fig. 3, therefore it seems that some non-ideal change in experimental system causes this oscillation. Robustness of the system is improved by choosing suitable observer gain.

In Fig. 10, the stator resistance $R_{s}^{*}$ of the controller is set to $2.3 \Omega$. The actual stator resistance $R_{s}$ is set to $1.6 \Omega$ in the simulation. The system is very near to unstable region and sustained oscillation is observed after the speed is changed. This result is well predicted by the trajectories of the poles

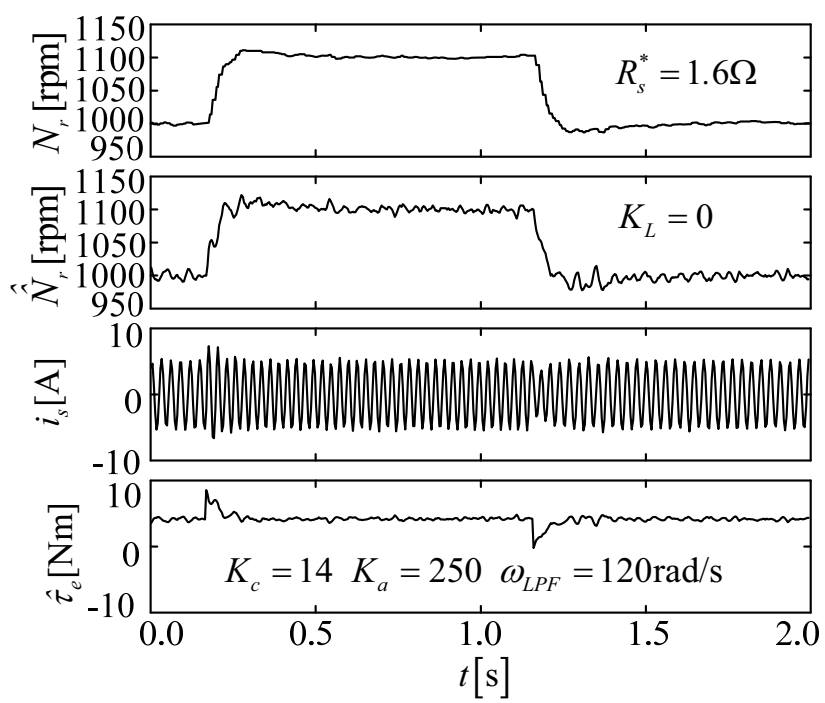

Fig.5. Step response without phase current low-pass filter, when $K_{a}=250, K_{c}=14, \omega_{L P F}=120, R_{s}^{*}=1.6 \Omega$ and with voltage compensation (experiment).

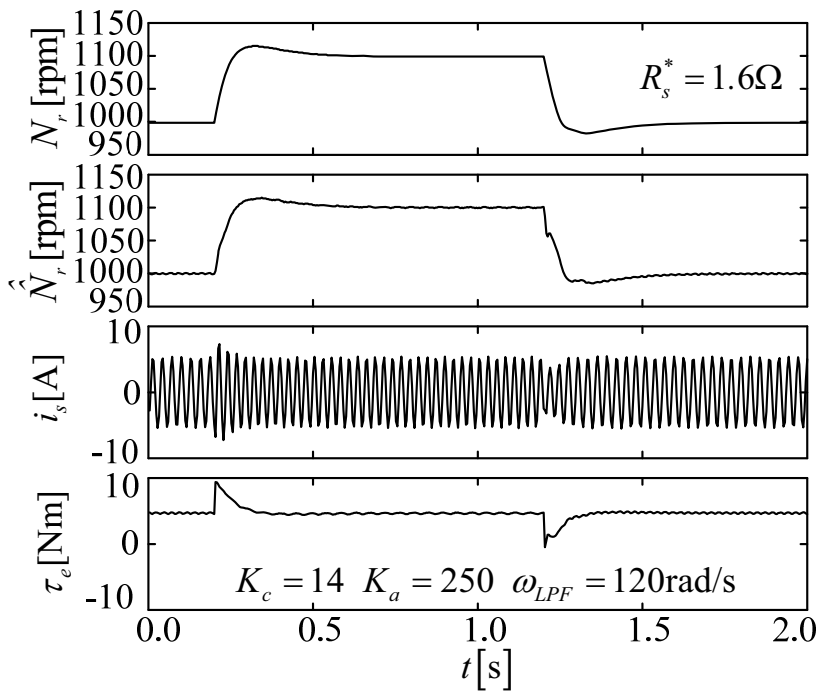

(a) Simulation

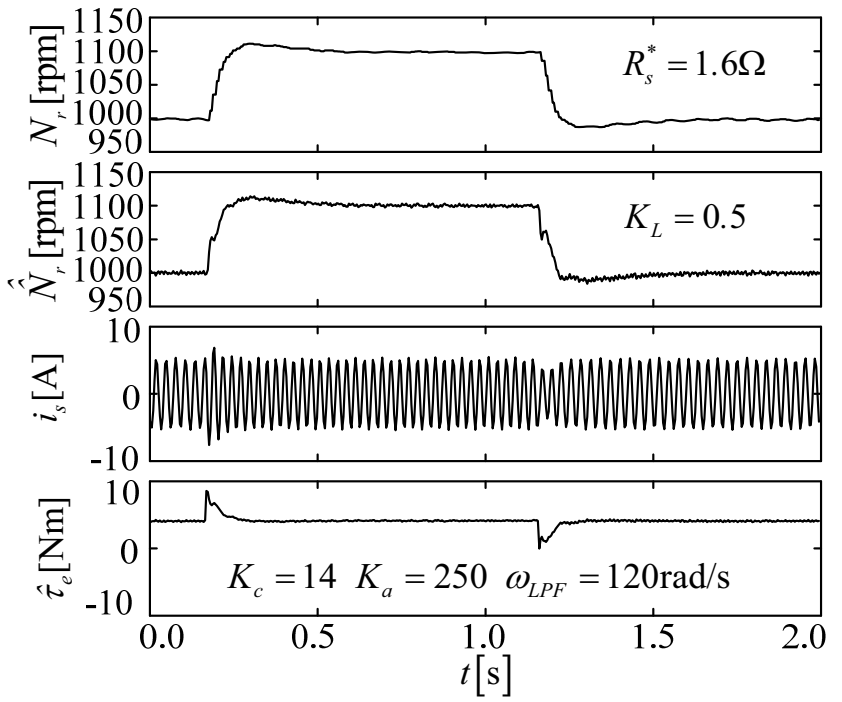

(b) Experiment

Fig.6. Step responses without voltage compensation. 


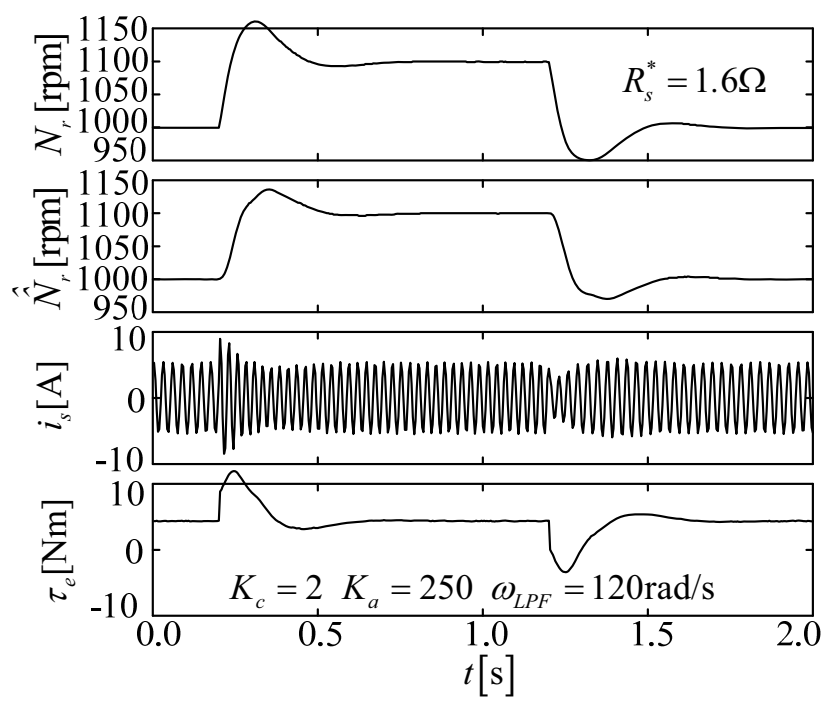

(a) Simulation.

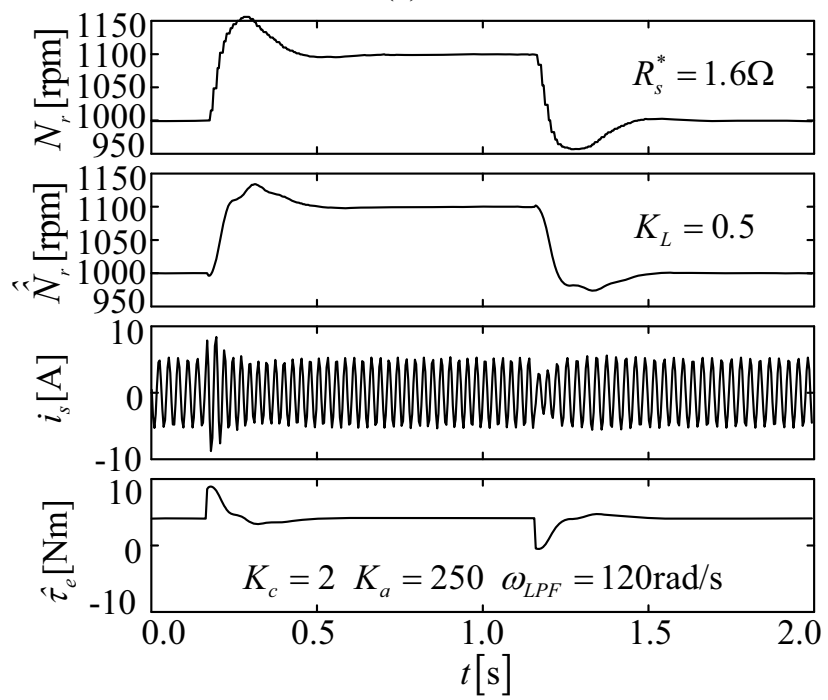

(b) Experiment

Fig.7. Step responses when $K_{c}$ is small.

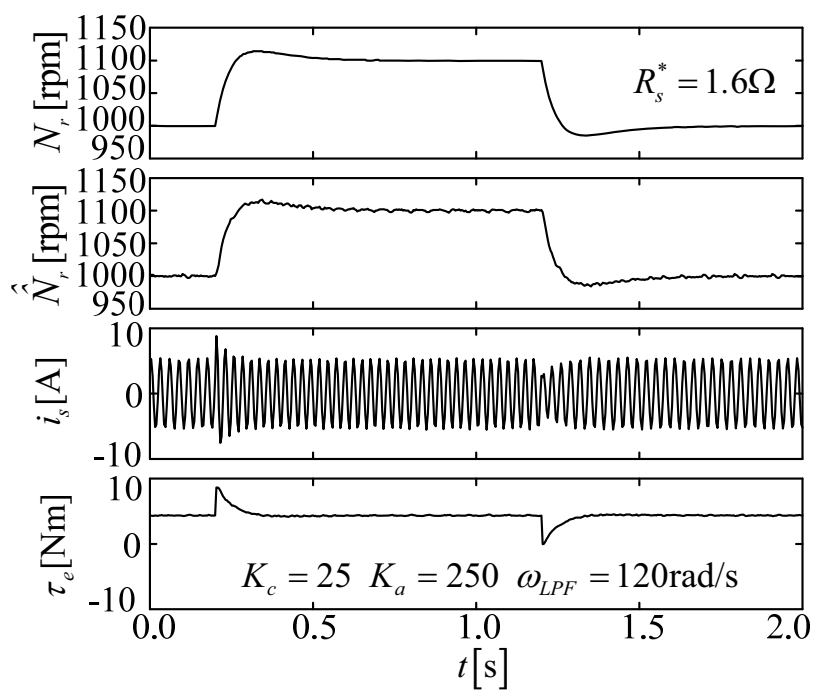

(a) Simulation

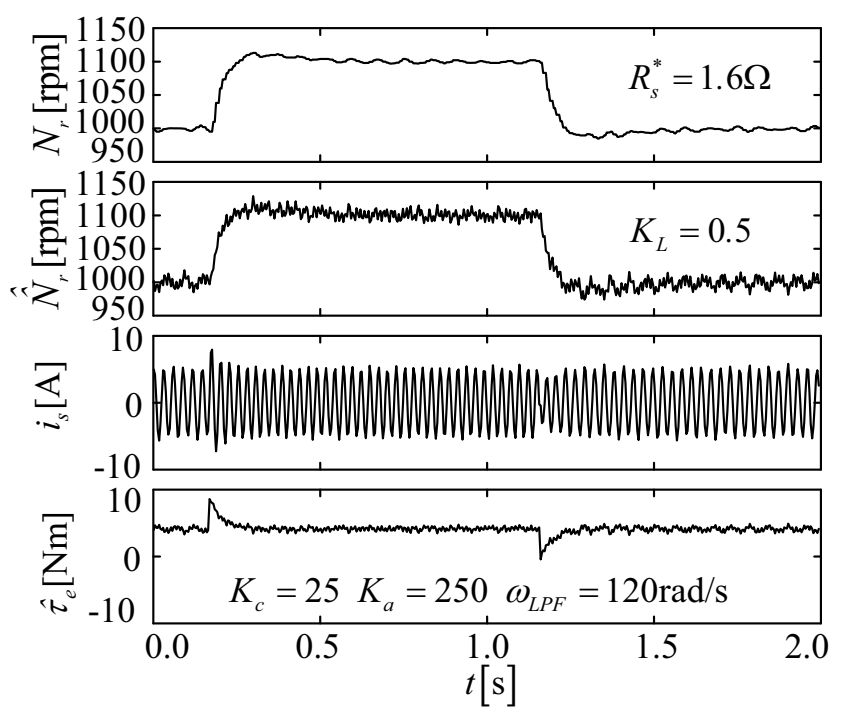

(b) Experiment

Fig.8. Step responses when $K_{c}$ is large.

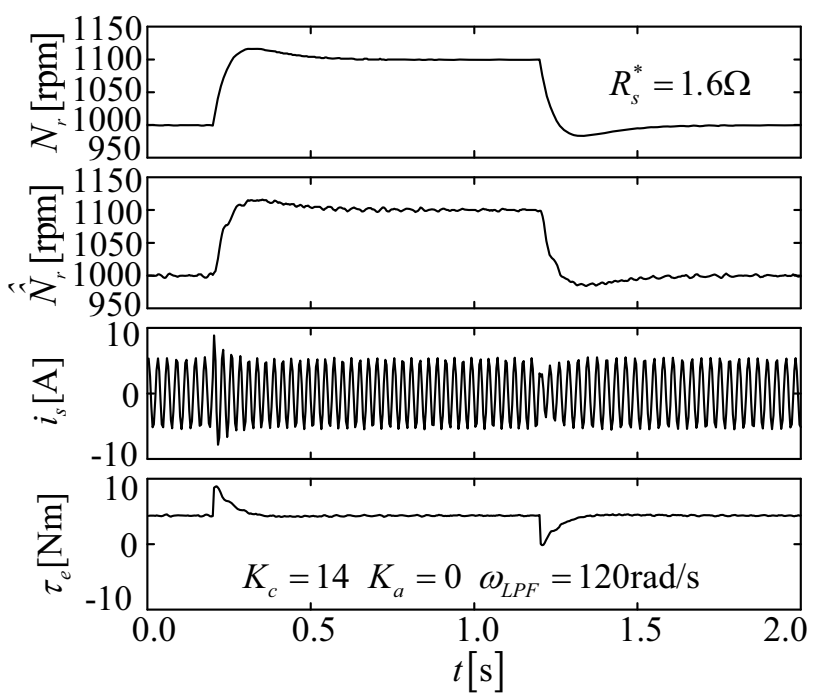

(a) Simulation

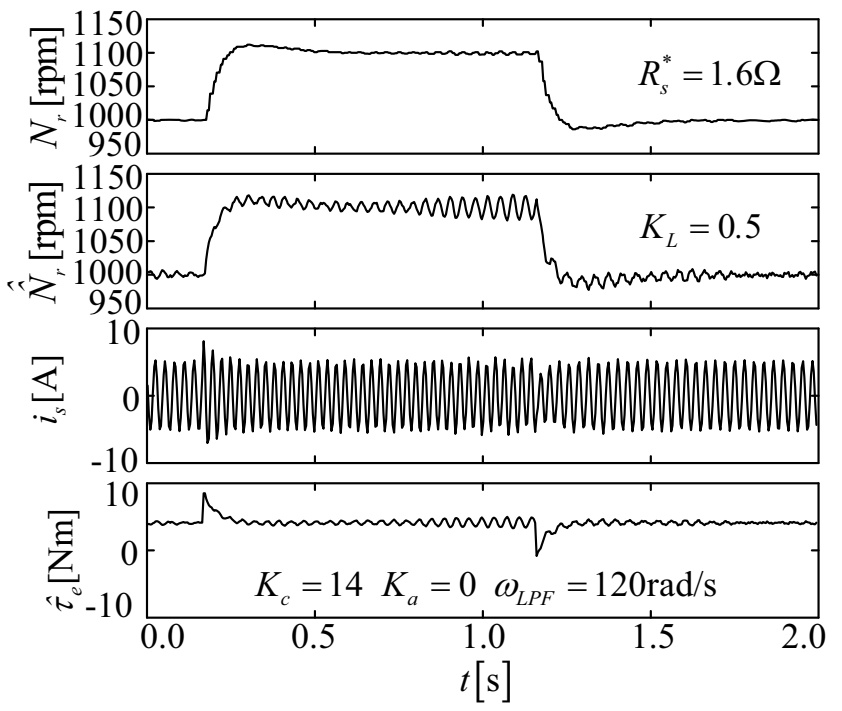

(b) Experiment

Fig.9. Step responses when observer gain is zero. 


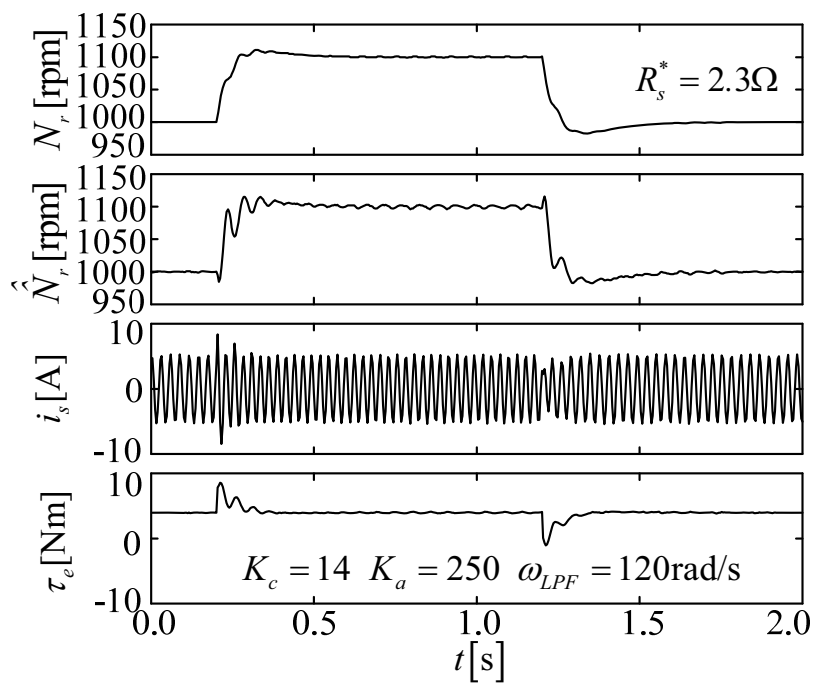

(a) Simulation

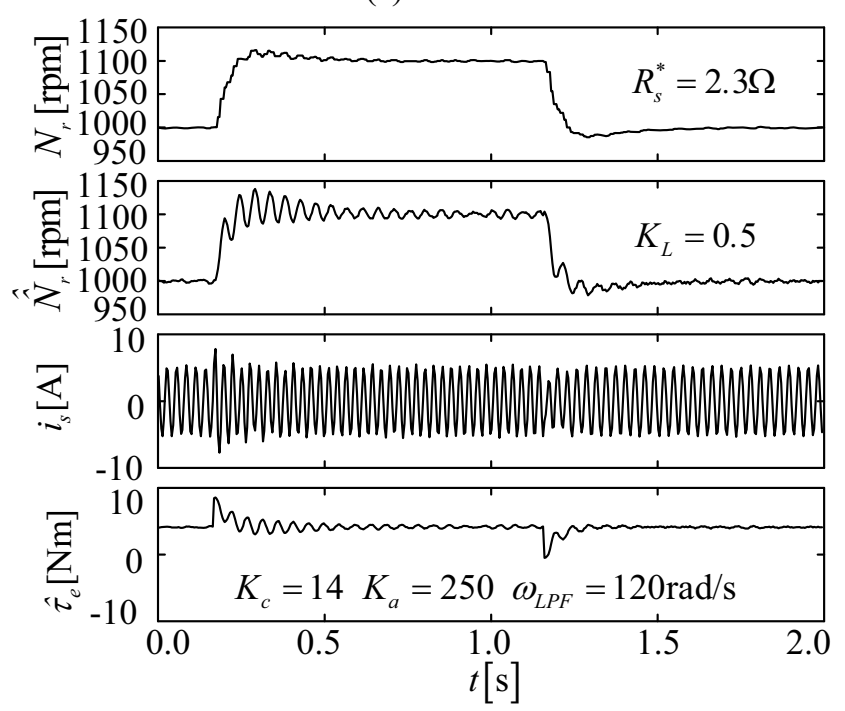

(b) Experiment

Fig.10. Step responses when stator resistance in controller is large.

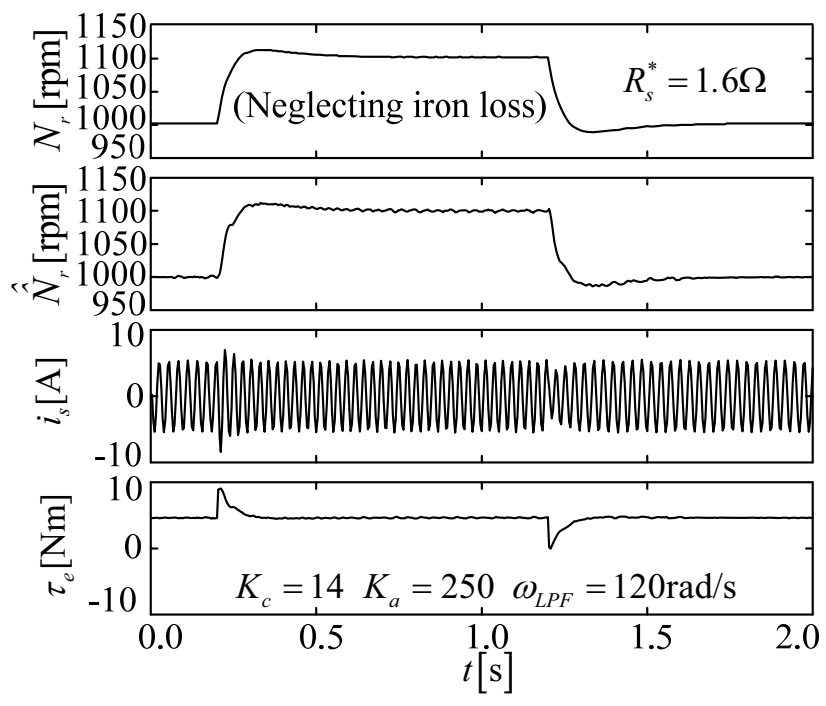

(a) Simulation

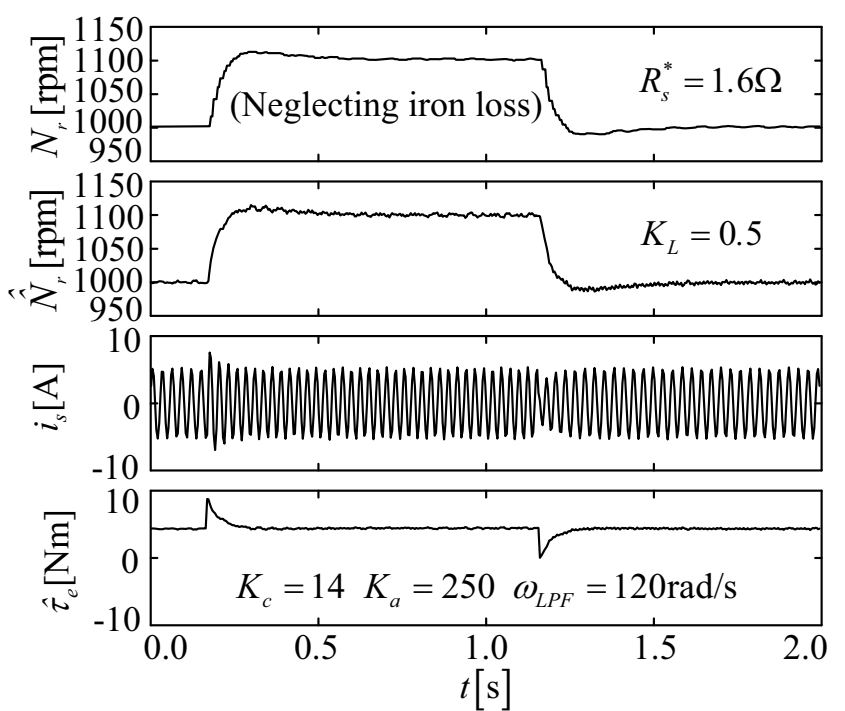

(b) Experiment

Fig.11 Step responses when iron loss is neglected.

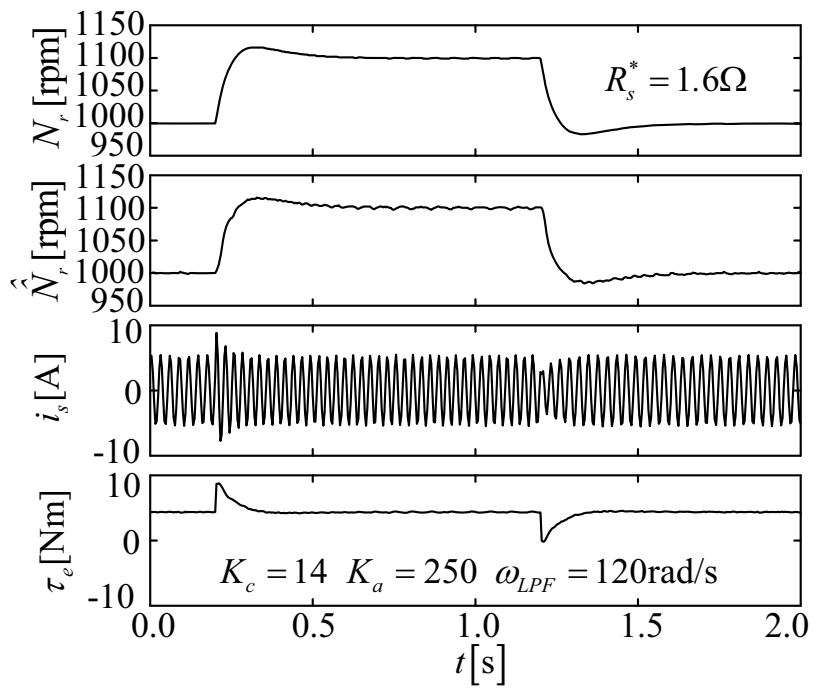

(a) Simulation

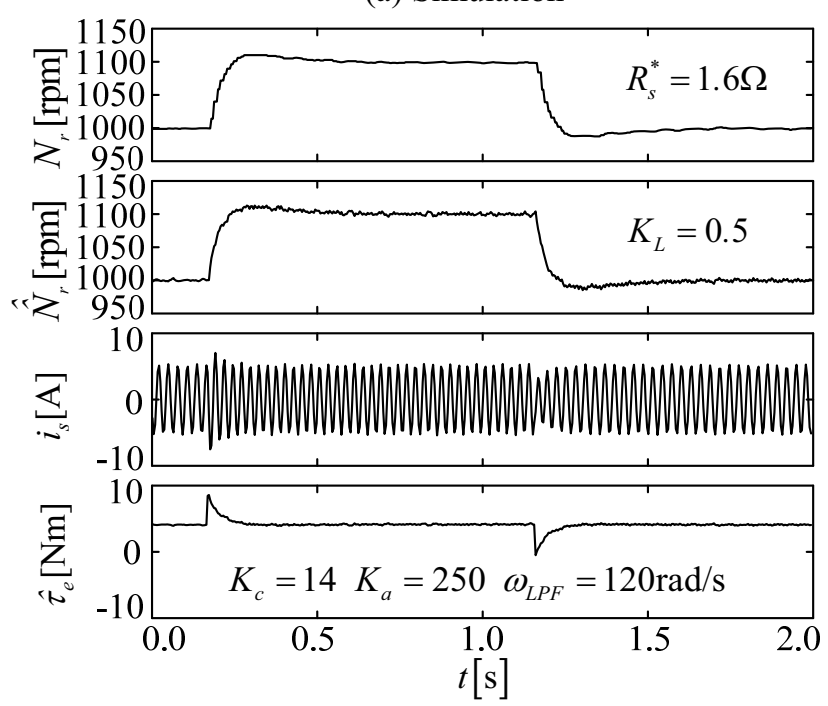

(b) Experiment

Fig.12. Step responses when $K_{c}=250, K_{c}=14$, $\omega_{L P F}=120, R_{s}^{*}=1.6 \Omega$ (designed parameters) 
and zeros shown in Fig.4. Simulated results are very close to the experimental ones.

Figure 11 shows the transient responses when the iron loss is neglected by setting very large $R_{m}^{*}$. According to the step response, we cannot observe the influence of the iron loss so greatly. This result is predicted by root locus shown in Fig.4. However, there are differences about steady state estimated torque. The estimated torque is almost equal to the load torque when the iron loss is considered.

The results obtained by designed control parameters such as $K_{a}=250, K_{c}=14, \omega_{L P F}=120, R_{s}^{*}=1.6 \Omega$ are shown in Fig.12. Both simulation and experimental results are almost same and good transient responses are obtained.

Figures 13 (a) and (b) show the simulation and experimental results respectively when the stator resistance identification is executed by (8). At first we set $R_{s}^{*}$ to $2.5 \Omega$.The identification is started at $0.6 \mathrm{~s}$. It is estimated that $R_{s}^{*}$ converges to its actual value and the oscillation is improved.
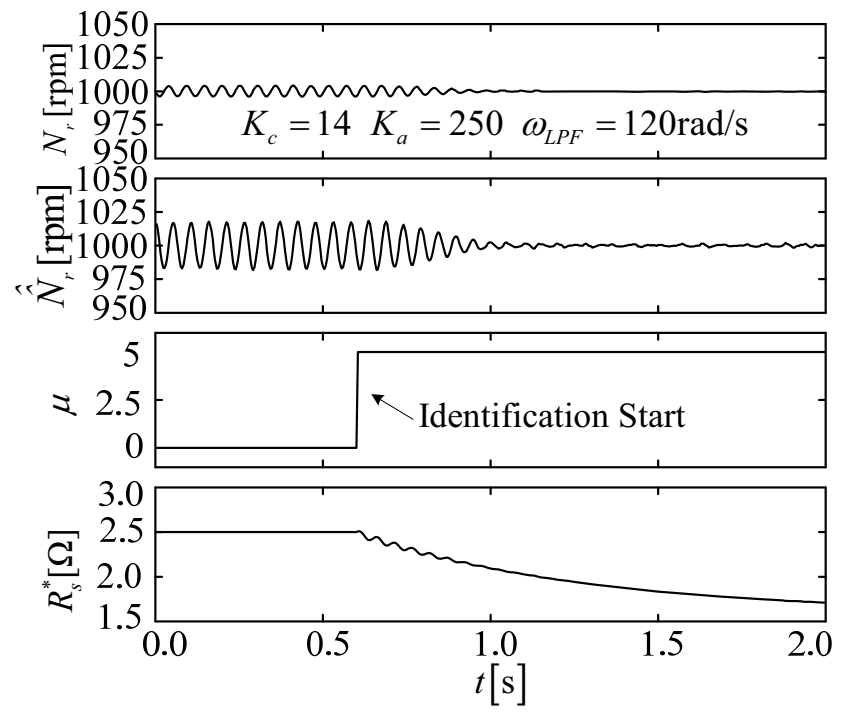

(a) Simulation

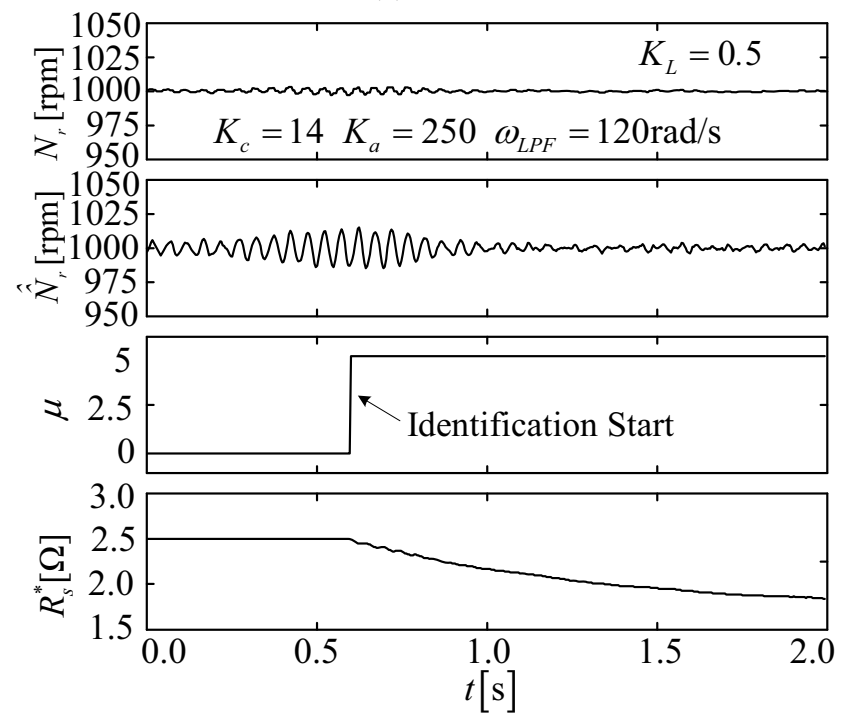

(b) Experiment

Fig.13. Step responses for the stator resistance identification.

\section{CONCLUSIONS}

In this paper, we have reported on the characteristic improvement of a novel current observer based induction motor speed sensorless system. The following conclusions are obtained.

(1) The proposed sensorless system is composed in a synchronously rotating reference frame and iron loss compensation and stator resistance identification are considered.

(2) A linear state equation is derived to study the stability of the system taking into account the iron loss, change of stator resistance and current observer gain. These effects are discussed by the trajectories of poles and zeros.

(3) Transient responses including stability limit are investigated by the digital simulation, which takes into account PWM mechanism in detail. These results agree well with the experimental results by introducing a low-pass filter for detecting stator current.

(4) Iron loss compensation is effective to estimate load torque but does not influence the transient characteristics.

(5) When the current observer gain $K_{a}$ is set to 0, large oscillation is observed in the experimental result. However, this oscillation is not confirmed by the simulation. Robustness of the system is improved by choosing suitable observer gain.

\section{REFERENCES}

[1] C. Schauder, "Adaptive Speed Identification for Vector Control of Induction Motors without Rotational Transducers", IEEE Trans. Industry Applic., vol.28, pp.1054-1061,1992.

[2] H. Kubota, K. Matsuse, "Speed sensorless field oriented control of induction machines using flux observer", in Proc. IECON’94, pp.1611-1615, 1994.

[3] M. Tsuji, E. Yamada, “ Advanced Vector Control for Induction Motor Drives", Proc. of Speedam, A1-1-7, 1998.

[4] M. Tsuji, S.Chen, K. Izumi, T,Ohta, E. Yamada, "A Speed Sensorless Induction Motor Vector Control System Using q-axis Flux with Parameter Identification", Conf. Rec, IEEE-IECON, pp.960-965,1997.

[5] M. Tsuji, S. Chen, K. Izumi, E. Yamada, "A Sensorless Vector Control System for Induction Motors Using q-Axis Flux with Stator Resistance Identification", IEEE Trans. Ind. Electron. Vol.48, No.1, pp.185-194, 2001.

[6] M. Tsuji, Y. Umesaki, R. Nakayama and K. Izumi, "A simplified MRAS based Sensorless Vector Control method of Induction Motor", Proc. PCC OSAKA, pp.1090-1095, 2002. 